\title{
Sexual function in older adults following thoracolumbar to pelvic instrumentation for spinal deformity
}

\author{
Clinical article
}

\author{
D. Kojo Hamilton, M.D., ${ }^{1}$ Justin S. Smith, M.D., Ph.D., ${ }^{2}$ \\ Tanya Nguyen, A.R.N.P., M.S.N., R.N., ${ }^{2}$ Vincent Arlet, M.D. ${ }^{3}$ \\ Manish K. Kasliwal, M.D., M.CH., ${ }^{2}$ And Christopher I. Shaffrey, M.D. ${ }^{2}$ \\ ${ }^{1}$ Department of Neurosurgery, Oregon Health \& Science University, Portland, Oregon; \\ ${ }^{2}$ Department of Neurosurgery, University of Virginia Health System, Charlottesville, Virginia; \\ and ${ }^{3}$ Department of Orthopedic Surgery, University of Pennsylvania, Philadelphia, Pennsylvania
}

\begin{abstract}
Object. Sexual function is an often-overlooked aspect of health-related quality of life among older adults treated for spinal deformity. The authors' objective was to assess sexual function among older adults following thoracolumbar fusion with pelvic fixation for spinal deformity.

Methods. This was a retrospective review of consecutive older adults ( $\geq 50$ years) treated with posterior thoracolumbar instrumentation (including pelvic fixation) for spinal deformity and with a minimum 18-month follow-up. Patients completed the Changes in Sexual Function Questionnaire-14 (CSFQ-14), Oswestry Disability Index (ODI), and 12-Item Short-Form Health Survey (SF-12).

Results. Sixty-two patients (45 women and 17 men) with a mean age of 70 years (range 50-83 years) met the inclusion criteria. Eight women did not complete all questionnaires and were excluded from the subanalysis. The mean number of instrumented levels was 9.8 (range 6-18), and the mean follow-up was 36 months (range 19-69 months). Based on the CSFQ-14, 13 patients (24\%) had normal sexual function, and 8 (15\%), 10 (19\%), and $23(42 \%)$ had mild, moderate, and severe dysfunction, respectively. Thirty-nine percent of patients reporting severe sexual dysfunction did not have available partners $-23 \%$ because of a partner's death and $16 \%$ because of a partner's illness) - or had significant medical comorbidities of their own (48\%). Thirty-nine percent of assessed patients had either no or only mild sexual dysfunction. Patients with minimal or mild disability tended to have no or mild sexual dysfunction.

Conclusions. The authors of this study assessed sexual function in older adults following surgical correction of spinal deformity that included posterior instrumented fusion and iliac bolts. Nearly $40 \%$ of assessed patients had either no or only mild sexual dysfunction, suggesting that despite an older age and extensive spinopelvic instrumentation, it remains very possible to maintain or achieve satisfactory sexual function.

(http://thejns.org/doi/abs/10.3171/2013.4.SPINE121078)
\end{abstract}

\begin{tabular}{|c|c|c|}
\hline \multicolumn{2}{|c|}{ KEY WORDS $\quad \bullet \quad$ adu } & - scoliosis - sexual function $•$ spine \\
\hline
\end{tabular}

A SUBSTANTIAL shift in population demographics is underway in many developed countries, with the proportion of elderly expanding to unprecedented levels. For example, between the years 2000 and 2030, the number of individuals at least 65 years of age in the US is expected to double to more than 70 million. ${ }^{24}$ As these shifts occur, it will become increasingly important to better appreciate and effectively manage the medical and surgical conditions that commonly afflict the elderly.

According to Schwab et al., ${ }^{18}$ the prevalence of spinal

Abbreviations used in this paper: CSFQ-14 $=14$-Item Changes in Sexual Function Questionnaire; HRQOL = health-related quality of life; ODI = Oswestry Disability Index; SF-12 = 12-Item Short-Form Health Survey. deformity among the elderly may be as high as $68 \%$. Although the finding of spinal deformity in many adults may be simply incidental and require only education and follow-up, for others it can produce substantial pain and disability that warrants consideration of surgical treatment. ${ }^{1}$ Several recent reports have demonstrated the potential of surgical treatment for spinal deformity in adults to provide improvement of pain and overall HRQOL. ${ }^{6,19-21}$ Although sexual function is an important aspect of HRQOL, it is not well assessed by standard outcome measures commonly used for spinal deformity surgery in adults. In addition, there is a general lack of discussion regarding sexual func-

This article contains some figures that are displayed in color online but in black-and-white in the print edition. 
tion after spine surgery, which may result from a number of issues, including societal and personal inhibitions and relatively limited information available to the counseling physician.,24

Studies of sexual function and counseling have been reported in patients with chronic pain, diabetes, and cardiac disorders and after hip arthroplasty..$^{13,15,22,23}$ There have also been reports of improved sexual function after the successful treatment of lumbar disc herniation and chronic back pain. 2,5,9,10 Moreover, sexual dysfunction has been reported in up to two-thirds of patients with back pain. ${ }^{16}$ Despite the potentially substantial impact of spinal deformity on HRQOL among the elderly, its impact on sexual function remains relatively unexplored.

Sexual function among the elderly can be affected by several conditions, including postmenopausal hypoactive sexual desire disorder, therapy for prostate cancer (androgen deprivation therapy), diabetic neuropathy, obesity, and poor cardiovascular health. Nevertheless, current literature seems to suggest that many older and elderly adults remain sexually active. A survey-based study by Lindau et al. ${ }^{14}$ documenting the prevalence of sexual activity among older adults in the US revealed that $73 \%$ of respondents in the 57- to 64-year age group are sexually active and that more than $50 \%$ of respondents in the 65 - to 74-year age group have an active sexual life.

Given the rapidly expanding elderly population, the high prevalence of spinal deformity among the elderly, and the relative lack of information regarding scoliosis surgery and sexual function, our objective in the present study was to assess sexual function among older adults following thoracolumbar to pelvic fixation for spinal deformity.

\section{Methods}

This was a retrospective review of consecutive cases from a single surgeon at a tertiary care, academic medical center with a high volume of adult patients with scoliosis. Surgeon case logs were reviewed for patients meeting the following inclusion criteria: age $\geq 50$ years, diagnosis of spinal deformity, surgical treatment with posterior thoracolumbar instrumentation (including pelvic fixation), and a minimum of 18 months of postoperative follow-up. Patients with underlying neurological or neuromuscular conditions were excluded.

After obtaining institutional review board approval to conduct the study, we approached consecutive patients either by telephone or at the time of routine clinical followup to participate in our study. Enrolled patients completed the CSFQ-14 12 for men and women and the ODI standardized questionnaire. The CSFQ-14 includes 14 questions that assess sexual functioning based on a 5-point Likert scale, with higher scores reflecting higher sexual functioning. The ODI is scored from 0 to 100 , with higher scores reflecting greater disability. For an additional global health assessment, patients also completed the SF-12. Moreover, any discomfort related to the iliac bolts when patients laid on their backs or while sitting in general was recorded. Patient demographics, comorbidities, and surgical procedure details were collected from medical and surgical records.

The potential impact of medical comorbidities, part- ner availability, and sex differences was assessed with regard to sexual function. Correlations between sexual function and outcome scores, using both the SF-12 and the ODI, were assessed. Statistical comparisons were performed using the Fisher exact test, with $\mathrm{p}<0.05$ considered statistically significant. All statistical comparisons were 2-sided and were performed using commercially available software (SPSS, version 19.0; SPSS, Inc.). Values are expressed as the means \pm standard deviations, unless indicated otherwise.

\section{Results}

Sixty-two patients (45 women and 17 men) met the inclusion criteria and consented to participate in our study. The mean age of study participants was $70 \pm 8.1$ years (range 50-83 years). Surgical indications included scoliosis (23 patients), kyphosis (8 patients), and kyphoscoliosis (31 patients). The mean number of instrumented levels was $9.8 \pm 2.9$ (range 6-18), and the mean duration of followup at the time of study enrollment was $36 \pm 15$ months (range 19-69 months). Twenty-nine patients (47\%) had a history of prior lumbar spine surgery. One woman did not fully complete the questionnaires and was excluded from data analysis. Seven women completed all questionnaires except for the CSFQ-14 and were excluded from analyses that included this questionnaire.

Overall, women reported a higher desire component on the CSFQ-14 than men $(p=0.031)$. Of the 54 patients who completed the CSFQ-14, 13 (24\%) had no sexual dysfunction and $8(15 \%), 10(19 \%)$, and $23(42 \%)$ had mild, moderate, and severe dysfunction, respectively (Fig. 1). Of the patients reporting severe sexual dysfunction, 9 (39\%) did not have an available partner ( $23 \%$ because of a partner's death and $16 \%$ because of a partner's illness), and medical comorbidities and resultant physical disability precluded another $11(48 \%)$ from reporting satisfactory sexual function.

Table 1 shows the distribution of sexual dysfunction category (none, mild, moderate, and severe) in relation to the severity of disability (minimal, moderate, severe, crippled, bed bound). Patients with minimal or moderate disability tended to have no or mild sexual dysfunction, as seen in the scatter plot in Fig. 2. Figures 3 and 4 feature scatter plots illustrating the relationship between disability (ODI) and sexual dysfunction (CSFQ-14) for men and women, respectively.

Patients who had above-average SF-12 scores on both the physical and mental components tended to have minimal or moderate disability according to the ODI (Tables 2 and 3; $\mathrm{p}<0.0001)$. One woman, who scored above average in both components of the SF-12 and had minimal disability, reported discomfort due to her iliac bolts and indicated that she was "mechanically unable to achieve orgasm with her long-term partner since extension of her fusion." No other patient reported that the iliac bolts caused discomfort or were "bothersome" with regard to sexual function. A history of prior major spine surgery did not have a significant impact on sexual dysfunction $(p>0.05)$.

The potential impact of patient age on sexual dysfunction was explored by dividing patients into 3 age groups 


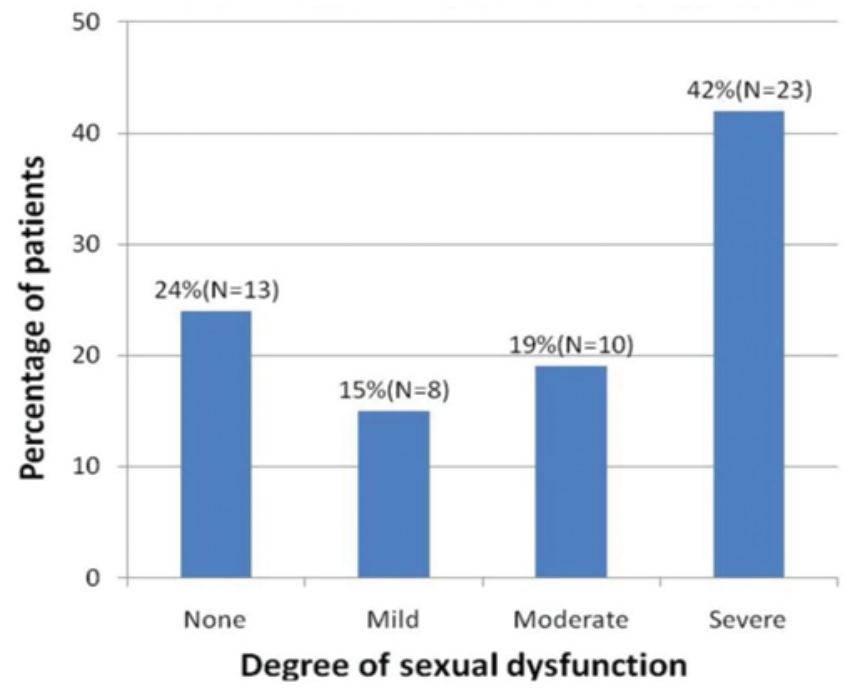

Fig. 1. Sexual dysfunction in 54 older adults after surgery for spinal deformity that included posterior thoracolumbar instrumented fusion and pelvic fixation. Sexual function was assessed using the CSFQ-14.

(50-59, 60-69, and 70-80 years), including only those without substantial comorbidities and those with a sexual partner. No significant differences in the individual components of the CSFQ-14 (that is, pleasure, desire/arousal, desire/interest, arousal/excitement, and orgasm/completion) were identified based on patient age group $(\mathrm{p}>0.05)$. Among patients in the 8th decade of life, there was a nonsignificant decrease in the orgasm/completion component $(p=0.17)$. Collectively, these analyses suggest that sexual dysfunction in this population may be less associated with age than with the absence of comorbidities or the lack of available partners.

\section{Discussion}

In this study we assessed sexual function in older adults following surgical correction of a spinal deformity that included posterior thoracolumbar instrumented fusion and iliac bolts. Findings demonstrated an overall high percentage of patients $(42 \%)$ with severe sexual dysfunction. However, for most of the patients who had disrupted

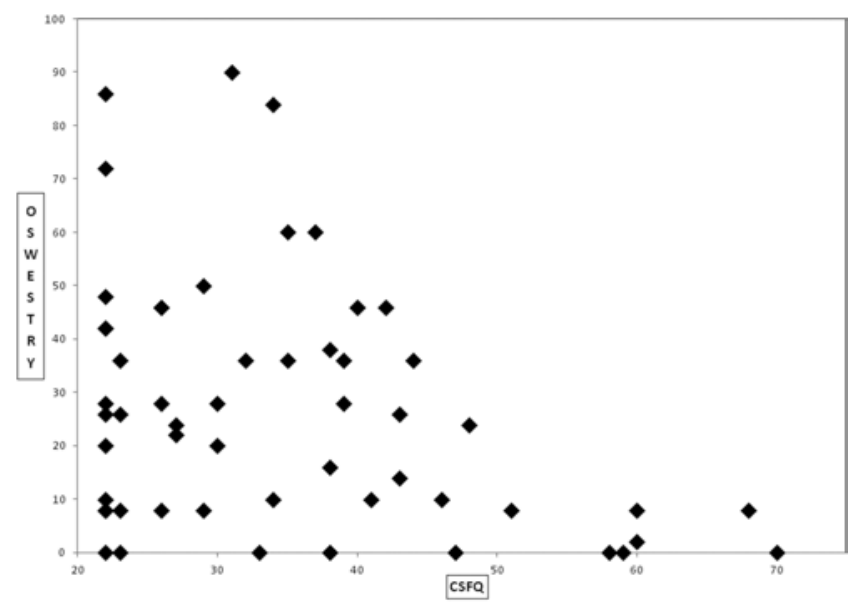

FIG. 2. Scatter plot illustrating the relationship between disability and sexual dysfunction in 54 older adults after surgery for spinal deformity that included posterior thoracolumbar instrumented fusion and pelvic fixation. Disability was assessed using the ODI, with higher scores reflecting greater disability. Sexual dysfunction was assessed using the CSFQ-14, with higher scores reflecting better sexual functioning. Note that several points overlap.

sexual function, it was attributable to the unavailability of partners (23\% because of a partner's death and $16 \%$ because of a partner's illness) or to medical comorbidities. Nearly $40 \%$ of assessed patients had either no or only mild sexual dysfunction, suggesting that despite an older age and extensive thoracolumbar spinal instrumentation with pelvic fixation, it is very possible to maintain or achieve satisfactory sexual function.

Sexuality and sexual function in adults following major spine surgery is rarely discussed. Although there is a high prevalence of sexual dysfunction in patients with back pain ${ }^{16}$ the literature to aid in the discussion of preand postsurgery sexual dysfunction pertaining to major degenerative spinal conditions remains limited. The majority of available literature relates to spinal cord injury, ${ }^{3,4}$ lumbar disc herniation, ${ }^{2}$ and degenerative disc disease. ${ }^{5,9}$ Although these studies provide valuable information, it is difficult to apply the findings to an older population treated for spinal deformity with substantially more complex and invasive procedures. Notably, in several studies relating

TABLE 1: Observed frequencies for sexual dysfunction and disability in 61 patients treated with posterior thoracolumbar instrumentation for spinal deformity

\begin{tabular}{|c|c|c|c|c|c|c|}
\hline \multirow[b]{2}{*}{ Parameter } & \multicolumn{6}{|c|}{ ODI Category } \\
\hline & Minimal & Moderate & Severe & Crippled & Bed Bound & Total \\
\hline \multicolumn{7}{|l|}{ CSFQ-14 category } \\
\hline none & 9 & 3 & 1 & 0 & 0 & 13 \\
\hline mild & 3 & 2 & 2 & 1 & 0 & 8 \\
\hline moderate & 4 & 4 & 0 & 0 & 2 & 10 \\
\hline severe & 8 & 8 & 5 & 1 & 1 & 23 \\
\hline CSFQ-14 not completed & 6 & 0 & 0 & 1 & 0 & 7 \\
\hline total & 30 & 17 & 8 & 3 & 3 & 61 \\
\hline
\end{tabular}




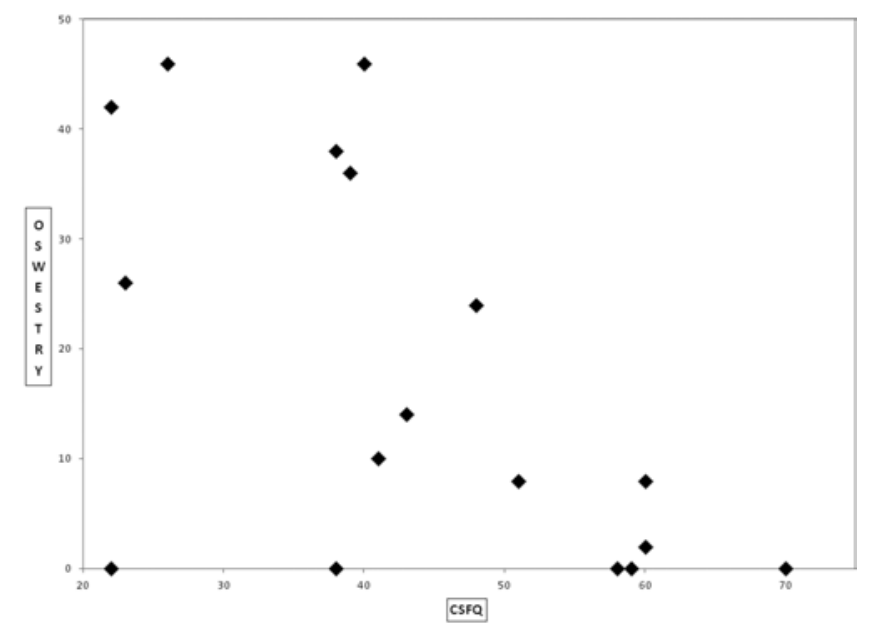

FIG. 3. Scatter plot illustrating the relationship between disability and sexual dysfunction in 17 older adult men after surgery for spinal deformity that included posterior thoracolumbar instrumented fusion and pelvic fixation. Disability was assessed using the ODI, with higher scores reflecting greater disability. Sexual dysfunction was assessed using the CSFQ-14, with higher scores reflecting better sexual functioning.

to sexual dysfunction in adults following spine surgery, a great impediment to research and education has been physician attitudes on the importance of the subject matter. ${ }^{2,24}$

To answer questions for patients and to inform surgeons, we used the CSFQ-14 to help elucidate sexual dysfunction in older adults following posterior instrumentation to the pelvis for spinal deformity. Components of the CSFQ-14 include pleasure, desire/arousal, desire/interest, arousal/excitement, and orgasm/completion. Women did score significantly high on desire components of the CSFQ-14; however, when taken in the context of the availability of partners and comorbid conditions, the overall

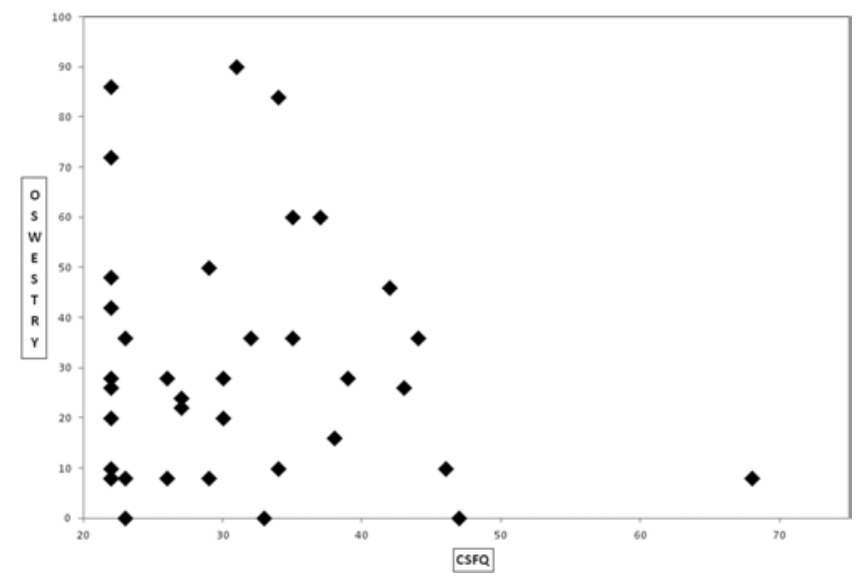

FIG. 4. Scatter plot illustrating the relationship between disability and sexual dysfunction in 37 older adult women after surgery for spinal deformity that included posterior thoracolumbar instrumented fusion and pelvic fixation. Disability was assessed using the ODI, with higher scores reflecting greater disability. Sexual dysfunction was based on the CSFQ-14, with higher scores reflecting better sexual functioning. Note that several points overlap.
TABLE 2: Relationship between disability and general physical health status for 61 older adults following posterior thoracolumbar instrumented fusion (including iliac bolts) for the treatment of spinal deformity*

\begin{tabular}{lccc}
\hline & \multicolumn{3}{c}{ SF-12 PCS } \\
\cline { 2 - 4 } ODI Category & Above Average & Below Average & Total \\
\hline minimal & 27 & 3 & 30 \\
moderate & 11 & 6 & 17 \\
severe & 3 & 5 & 8 \\
crippled & 1 & 2 & 3 \\
bed bound & 2 & 1 & 3 \\
total & 44 & 17 & 61 \\
\hline
\end{tabular}

* PCS = physical component score.

score was not significantly affected by the individual components. Not surprisingly, patients with greater disability generally had greater sexual dysfunction, and the degree of disability (ODI) was significantly negatively impacted by a poorer physical and mental health status (SF-12).

Contrary to what may be expected, the present study reveals a very low incidence of complaints related to iliac bolts. Only 1 of 61 patients in the present series had symptoms attributed to this instrumentation. This finding contrasts with previous reports that suggest higher rates of symptoms, occasionally severe enough to warrant surgical removal of the bolts. ${ }^{11,17}$ We believe that the incidence of symptoms may relate to the technique used for placement. We routinely seat the iliac screw heads below the surface of the posterior superior iliac spine by creating a small osteotomy in its medial wall. Low-profile lateral connectors are then used to link the iliac bolts to the rods.

Previous studies have assessed sexual function following the treatment of spinal conditions and are worth noting. A study by Danielsson et al..$^{8}$ on the sexual life of young adult women treated for adolescent idiopathic scoliosis showed that $33 \%$ of the surgically treated and $28 \%$ of those with a brace experienced reduced or limited sexual activities as compared with the control group, even though these young women with scoliosis had no neurological dysfunction. Berg et al..$^{5}$ demonstrated improvement in sexual satisfaction after surgery in patients with chronic low-back pain who had undergone a posterior lumbar fusion or total disc replacement, and this improvement correlated with a reduction in low-back pain after surgery. Akbaş et al. ${ }^{2}$ reported significant improvement in sexual life after surgery for lumbar disc herniation at the 2-month follow-up. In contrast, Kanayama et al.., ${ }^{10}$ in a recent study on sexual desire and activities following lumbar disc herniation surgery, showed that $46 \%$ of women still felt discomfort after surgery. The authors suggested that even patients with significant pain relief following surgery might require counseling, including a discussion on the timing of a return to sexual activity and the safety of various sexual positions to enhance satisfaction.

Although the present study represents a unique contribution to the literature, it is important to recognize its 
TABLE 3: Relationship between disability and general mental health status for 61 older adults following posterior thoracolumbar instrumented fusion (including iliac bolts) for the treatment of spinal deformity*

\begin{tabular}{lccc}
\hline & \multicolumn{3}{c}{ SF-12 MCS } \\
\cline { 2 - 4 } ODI Category & Above Average & Below Average & Total \\
\hline minimal & 23 & 7 & 30 \\
moderate & 10 & 7 & 17 \\
severe & 3 & 5 & 8 \\
crippled & 0 & 3 & 3 \\
bed bound & 0 & 3 & 3 \\
total & 36 & 25 & 61 \\
\hline
\end{tabular}

* MCS = mental component score.

limitations. The primary limitations are its retrospective design and the lack of preoperative assessment of sexual function. Other limitations include the heterogeneous patient population with a wide range of ages, spinal deformities, and comorbidities. However, this population reflects the heterogeneity encountered in an adult spinal deformity practice. The present report should be viewed as a basis for subsequent prospective controlled investigation, and efforts are currently underway to develop such a study.

\section{Conclusions}

Sexual function is an often overlooked, but important, aspect of HRQOL among older adults treated for spinal deformity. This study provides assessment of sexual function in older adults following surgical correction of spinal deformity that included posterior thoracolumbar instrumented fusion and iliac bolts. The findings demonstrate an overall high percentage $(42 \%)$ of patients with severe sexual dysfunction. However, for most of the patients, disrupted sexual function was attributable to the unavailability of a partner (23\% because of a partner's death and $16 \%$ because of a partner's illness) or to medical comorbidities. Nearly $40 \%$ of patients assessed had either no or only mild sexual dysfunction, suggesting that despite an older age and extensive thoracolumbar spinal instrumentation with pelvic fixation, it is very possible to maintain or achieve satisfactory sexual function.

\section{Disclosure}

Dr. Arlet is a consultant for DePuy Synthes and holds a patent with the University of Virginia. Dr. Smith is a consultant for Biomet, Medtronic, and DePuy and has received support from DePuy for non-study-related clinical or research effort. Dr. Shaffrey is a consultant for Biomet, Medtronic, Nuvasive, Globus, Stryker; has received royalties from Biomet; holds a patent with Medtronic; and has received clinical or research support from DePuy but not for the study described herein.

Author contributions to the study and manuscript preparation include the following. Conception and design: Smith, Hamilton, Shaffrey. Acquisition of data: Hamilton, Nguyen. Analysis and interpretation of data: Smith, Hamilton, Kasliwal. Drafting the article:
Smith, Hamilton, Kasliwal, Shaffrey. Critically revising the article: all authors. Reviewed submitted version of manuscript: all authors. Approved the final version of the manuscript on behalf of all authors: Smith. Statistical analysis: Hamilton. Study supervision: Shaffrey.

\section{References}

1. Aebi M: The adult scoliosis. Eur Spine J 14:925-948, 2005

2. Akbaş NB, Dalbayrak S, Külcü DG, Yilmaz M, Yilmaz T, Naderi S: Assessment of sexual dysfunction before and after surgery for lumbar disc herniation. Clinical article. J Neurosurg Spine 13:581-586, 2010

3. Alexander MS, Biering-Sørensen F, Elliott S, Kreuter M, Sønksen J: International spinal cord injury female sexual and reproductive function basic data set. Spinal Cord 49:787-790, 2011

4. Alexander MS, Rosen RC, Steinberg S, Symonds T, Haughie S, Hultling C: Sildenafil in women with sexual arousal disorder following spinal cord injury. Spinal Cord 49:273-279, 2011

5. Berg S, Fritzell P, Tropp H: Sex life and sexual function in men and women before and after total disc replacement compared with posterior lumbar fusion. Spine J 9:987-994, 2009

6. Bridwell KH, Glassman S, Horton W, Shaffrey C, Schwab F, Zebala LP, et al: Does treatment (nonoperative and operative) improve the two-year quality of life in patients with adult symptomatic lumbar scoliosis: a prospective multicenter evidencebased medicine study. Spine (Phila Pa 1976) 34:2171-2178, 2009

7. Clayton AH, Cantor-Cooke R: Satisfaction: Women, Sex, and the Quest for Intimacy. New York: Ballantine Books, 2007

8. Danielsson AJ, Nachemson AL: Childbearing, curve progression, and sexual function in women 22 years after treatment for adolescent idiopathic scoliosis: a case-control study. Spine (Phila Pa 1976) 26:1449-1456, 2001

9. Hägg O, Fritzell P, Nordwall A: Sexual function in men and women after anterior surgery for chronic low back pain. Eur Spine J 15:677-682, 2006

10. Kanayama M, Horio M, Umi Y, Yamaguchi A, Omata J, Togawa $\mathrm{D}$, et al: How does surgery affect sexual desire and activities in patients with lumbar disc herniation? Spine (Phila Pa 1976) 35:647-651, 2010

11. Kasten MD, Rao LA, Priest B: Long-term results of iliac wing fixation below extensive fusions in ambulatory adult patients with spinal disorders. J Spinal Disord Tech 23:e37-e42, 2010

12. Keller A, McGarvey EL, Clayton AH: Reliability and construct validity of the Changes in Sexual Functioning Questionnaire short-form (CSFQ-14). J Sex Marital Ther 32:43-52, 2006

13. Laffosse JM, Tricoire JL, Chiron P, Puget J: Sexual function before and after primary total hip arthroplasty. Joint Bone Spine 75:189-194, 2008

14. Lindau ST, Schumm LP, Laumann EO, Levinson W, O'Muircheartaigh CA, Waite LJ: A study of sexuality and health among older adults in the United States. N Engl J Med 357:762-774, 2007

15. Maigne JY, Chatellier G: Assessment of sexual activity in patients with back pain compared with patients with neck pain. Clin Orthop Relat Res (385):82-87, 2001

16. Maruta T, Osborne D: Sexual activity in chronic pain patients. Psychosomatics 19:531-537, 1978

17. O'Shaughnessy B A, Lenke LG, Bridwell KH, Cho W, Zebala LP, Chang MS, et al: Should symptomatic iliac screws be electively removed in adult spinal deformity patients fused to the sacrum? Spine (Phila Pa 1976) 37:1175-1181, 2012

18. Schwab F, Dubey A, Gamez L, El Fegoun AB, Hwang K, Pagala M, et al: Adult scoliosis: prevalence, SF-36, and nutritional parameters in an elderly volunteer population. Spine (Phila Pa 1976) 30:1082-1085, 2005

19. Smith JS, Shaffrey CI, Berven S, Glassman S, Hamill C, Horton $\mathrm{W}$, et al: Improvement of back pain with operative and non- 


\section{K. Hamilton et al.}

operative treatment in adults with scoliosis. Neurosurgery $\mathbf{6 5}$ : 86-94, 2009

20. Smith JS, Shaffrey CI, Berven S, Glassman S, Hamill C, Horton $\mathrm{W}$, et al: Operative versus nonoperative treatment of leg pain in adults with scoliosis: a retrospective review of a prospective multicenter database with two-year follow-up. Spine (Phila Pa 1976) 34:1693-1698, 2009

21. Smith JS, Shaffrey CI, Glassman SD, Berven SH, Schwab FJ, Hamill CL, et al: Risk-benefit assessment of surgery for adult scoliosis: an analysis based on patient age. Spine (Phila Pa 1976) 36:817-824, 2011

22. Spector IP, Leiblum SR, Carey MP, Rosen RC: Diabetes and female sexual function: a critical review. Ann Behav Med 15: 257-264, 1993

23. Steinke E, Patterson-Midgley P: Sexual counseling following acute myocardial infarction. Clin Nurs Res 5:462-472, 1996
24. US Census Bureau: Population Projections of the United States by Age, Sex, Race, and Hispanic Origin: 1995-2050. (http://www.census.gov/prod/1/pop/p25-1130.pdf) [Accessed April 15, 2013]

25. Yee L: Aging and sexuality. Aust Fam Physician 39:718-721, 2010

Manuscript submitted November 25, 2012.

Accepted April 11, 2013.

Please include this information when citing this paper: published online May 21, 2013; DOI: 10.3171/2013.4.SPINE121078.

Address correspondence to: Justin S. Smith, M.D., Ph.D., Department of Neurosurgery, University of Virginia, P.O. Box 800212, Charlottesville, Virginia 22908. email: jss7f@ virginia.edu. 